\title{
Comparison of Current Systemic Combination Therapies for Metastatic Hormone-Sensitive Prostate Cancer and Selection of Candidates for Optimal Treatment: A Systematic Review and Bayesian Network Meta-Analysis
}

OPEN ACCESS

Edited by:

Mohamed Saad Zaghloul,

Cairo University, Egypt

Reviewed by:

Gwenaelle Gravis, Institut Paoli-Calmettes (IPC), France

Adam R. Metwalli,

Howard University Hospital,

United States

*Correspondence:

Hao Zeng

kucaizeng@163.com

Pengfei Shen

cdhx510@163.com

tThese authors have contributed equally to this work and share co-first authorship

Specialty section

This article was submitted to Genitourinary Oncology,

a section of the journal

Frontiers in Oncology

Received: 11 December 2019

Accepted: 13 August 2020

Published: 18 September 2020

Citation:

Chen J, Ni Y, Sun G, Liao B, Zhang X, Zhao J, Zhu S, Wang Z, Shen $P$ and Zeng H (2020) Comparison of Current Systemic Combination Therapies for Metastatic Hormone-Sensitive

Prostate Cancer and Selection of Candidates for Optimal Treatment: A Systematic Review and Bayesian Network Meta-Analysis.

Front. Oncol. 10:519388. doi: 10.3389/fonc.2020.519388
Junru Chen ${ }^{\dagger}$, Yuchao $\mathrm{Ni}^{+}$, Guangxi Sun ${ }^{\dagger}$, Banghua Liao ${ }^{\dagger}$, Xingming Zhang, Jinge Zhao, Sha Zhu, Zhipeng Wang, Pengfei Shen* and Hao Zeng*

Department of Urology, Institute of Urology, West China Hospital, Sichuan University, Chengdu, China

Objective: To compare the efficacy and safety of current systemic combination therapies for patients with mHSPC and help select candidates for optimal treatment.

Methods: Databases of MEDLINE and EMBASE, Cochrane Central Register of Controlled Trials, and Clinical Trial.gov were searched for eligible studies. Direct and network meta-analysis were conducted to compare various systemic combination therapies and the surface under the cumulative ranking curve (SUCRA) was generated for treatment ranking. Subgroup analyses were performed according to the extent of metastasis. Adverse events (AEs) were compared among the effective treatments.

Results: Ten trials with 16 publications were included in this network meta-analysis. Direct and network meta-analysis consistently suggested that androgen-deprivation therapy (ADT) combined with docetaxel, abiraterone, enzalutamide, or apalutamide could significantly improve overall survival (OS) and failure-free survival (FFS) compared to ADT alone in men with mHSPC. SUCRA analysis demonstrated the superiority of ADT plus abiraterone or enzalutamide over other therapies. Subgroup analyses indicated that additional abiraterone to ADT had the highest ranking in patients with high-volume diseases or visceral metastases and enzalutamide plus ADT outperformed other treatments in patients with low-volume diseases or without visceral metastases. Different combination therapies had variable AE profiles and ADT in addition with docetaxel or abiraterone had the highest risk of AEs.

Conclusion: ADT plus docetaxel, abiraterone, enzalutamide, or apalutamide were associated with significantly improved survival in patients with mHSPC. ADT plus abiraterone or enzalutamide appeared to be the most effective treatments. Clinicians should balance the efficacy, potential AEs, and disease status to select the optimal treatment.

Keywords: metastatic prostate cancer, combination therapy, network meta-analysis, chemotherapy, androgen-deprivation therapy 


\section{INTRODUCTION}

Prostate cancer (PCa) remains the most frequent solid malignancy among men in the United States, accounting for $20 \%$ of the new cancer cases in 2019 (1). Approximately of $5 \%$ patients diagnosed as PCa have distant metastases with a 5 -year relative survival rate of only $30 \%$ (1). Over the past few decades, the standard of care for metastatic hormone-sensitive prostate cancer (mHSPC) has been androgen-deprivation therapy (ADT) through either surgical or medical castration. Unfortunately, almost all of the patients treated with ADT, or even ADT plus first-generation antiandrogen, ultimately become castration-resistant in a short time (2). It has been reported that no improvement in survival for men with mHSPC was observed for the last 20 years (3). Several randomized controlled trials (RCTs) have investigated the effectiveness of combination therapy in patients with mHSPC and suggested improved survival with additional bisphosphonates, celecoxib, docetaxel, or abiraterone alone or in combination (4-15). Therefore, the question arises spontaneously which is the optimal systemic treatment for men with mHSPC. Previous studies conducting comprehensive analyses demonstrated that abiraterone plus ADT might outperform other regimens in terms of survival $(16,17)$. However, recently, RCTs exploring the efficacy of ADT in addition to novel androgen receptor (AR) axis inhibitors such as enzalutamide and apalutamide, have emerged (18-20). To date, data regarding head-to-head comparisons of these combination therapies are limited. Thus, we conducted this updated systematic review and network meta-analysis aiming to compare the efficacy and safety of current combination systemic therapies for patients with $\mathrm{mHSPC}$ and help select candidates for optimal treatment.

\section{METHODS}

\section{Protocol and Registration}

We developed a protocol defining search strategy and data analysis for this review, in accordance with the Preferred Reporting Items for Systematic Reviews and Meta-analyses (PRISMA) statement (21). The full protocol of this review was registered on PROSPERO (ID: CRD42019132967).

\section{Search Strategy}

Databases of MEDLINE (1966-2019.9) and EMBASE (19742019.9) were searched through OVIDSP. Further searches were conducted through Cochrane Central Register of Controlled Trials (CENTRAL) (1948-2019.9) and Clinical Trial.gov (19992019.9). In addition, references of the included trials were screened for extra eligible trials. There was no restriction on language. The full search strategy was available in protocol and the last search was conducted on Sep 25, 2019.

\section{Eligibility and Exclusion Criteria}

The eligibility criteria were (1) RCTs comparing systemic treatments for patients with mHSPC; (2) trials reporting survival outcomes. Exclusion criteria consisted of (1) patients with castration-resistant prostate cancer (CRPC); (2) patients without metastatic diseases; (3) cohorts, reviews, or case reports.

\section{Outcomes}

The primary outcome was overall survival (OS), defined as the time from randomization to death from any cause. The secondary outcomes were failure-free survival (FFS) and adverse events (AEs). FFS was defined as the time from randomization until biological, radiographic/clinical progression, or death. For AEs, only AEs $\geq$ grade 3 were recorded to compare safety.

\section{Study Selection and Data Extraction}

Two reviewers (JRC and YCN) independently examined the titles and abstracts of the publications meeting the eligibility criteria. For potentially eligible studies, full texts were assessed to identify the final included trials. Any discrepancies were reconciled by discussion or a third review (GXS). For included studies, we extracted information as follows: recruitment period, study population, follow-up time, number of patients, treatment comparisons, the definition of outcomes, performance status, patients age, prostate-specific antigen (PSA) value, $\mathrm{T}$ and $\mathrm{N}$ category, location of metastases, total Gleason score, disease volume, and type and incidence of AEs $\geq$ grade 3 . We also collected the hazard ratio (HR) and corresponding 95\% confidence interval (95\% CI) for survival outcomes. For trials regarding enzalutamide and apalutamide, data in patients without prior docetaxel was used for analysis when it was available.

\section{Risk of Bias Assessment}

Two reviewers evaluated the risk of bias of the included studies using RevMan software (version 5.3) based on Cochrane Handbook. The following items were assessed: (1) random sequence generation; (2) allocation concealment; (3) blinding of participants and personnel; (4) blinding of outcome assessment; (5) incomplete outcome data; (6) selective reporting; and (7) other sources of bias.

\section{Statistical Analysis}

Direct comparisons were performed using RevMan 5.3. Fixedeffect model or random-effect model was used according to heterogeneity. Heterogeneity among trials in each comparison was evaluated with $I^{2}$ statistic and $\chi^{2}$ test and $I^{2}>50 \%$ or $\chi^{2} p<0.1$ was considered as significant heterogeneity. The Bayesian network meta-analysis was conducted using Gemtc package within R program. Effect size for the Bayesian network meta-analysis was presented with 95\% credible interval (CrI), as CrI was a more appropriate index than CI in Bayesian mixed treatment comparisons. Treatment rank probability was calculated and the surface under the cumulative ranking curve (SUCRA) was generated to describe the re-scaled mean ranking. SUCRA would be 1 if a treatment is certainly to be the best, whereas a treatment always ranks last would have a SUCRA value of 0 (22). Subgroup analyses for the most effective treatments were conducted according to disease volume and absence of visceral metastasis. High-volume disease was defined as the presence of visceral metastases or $\geq 4$ bone lesions with $\geq 1$ 


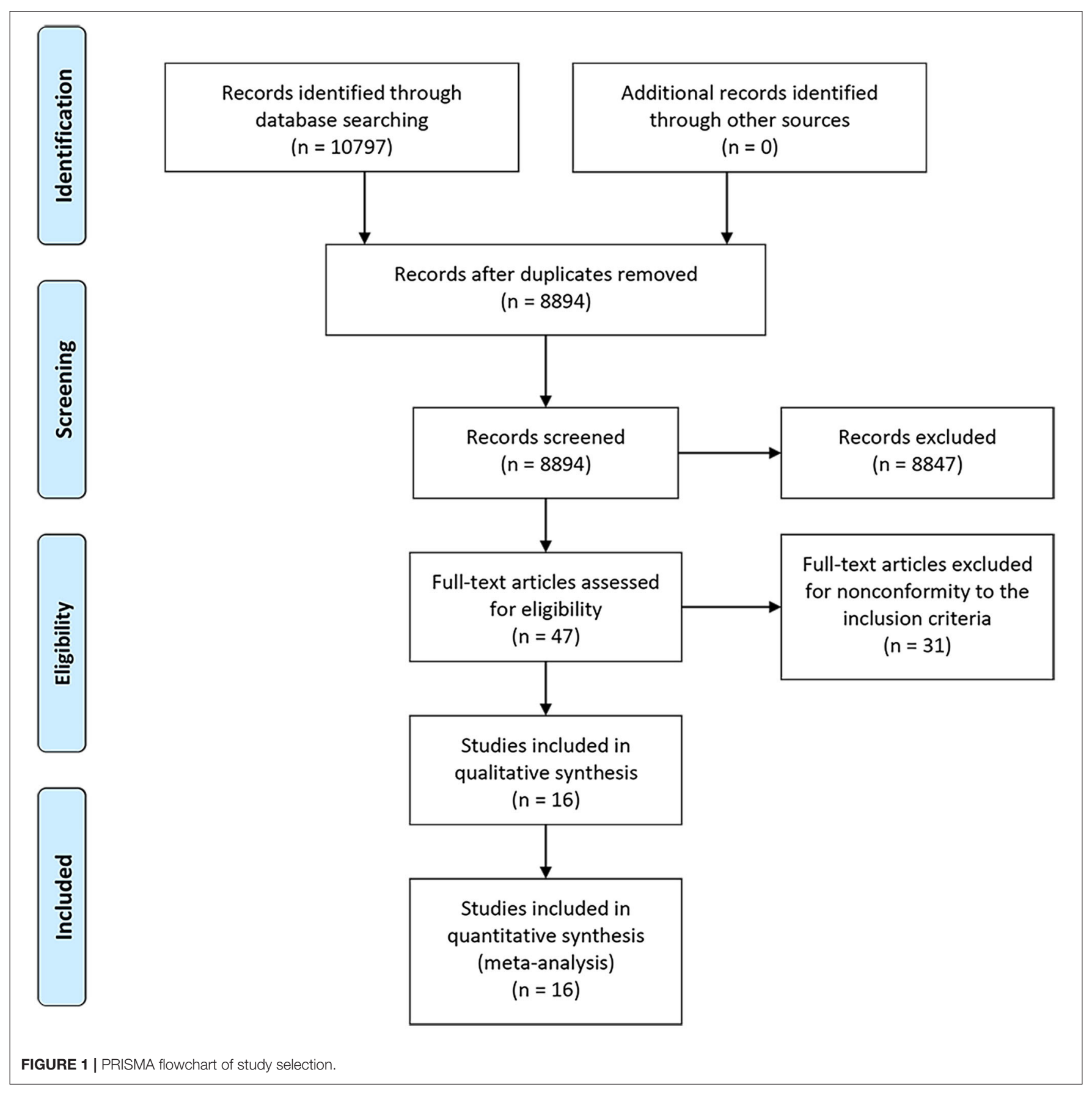

beyond the vertebral bodies and pelvis based on CHAARTED criteria (14). For trials with multiple reports, only the latest outcome data were used for analysis.

\section{RESULTS}

\section{Characteristics of Included Trials and Patients}

A PRISMA flowchart summarizing the selection process of the eligible studies was shown in Figure 1. In total, 10 RCTs with
16 full-text articles and 11174 patients were included in the final quantitative meta-analysis $(4-15,18-20,23)$. Characteristics of the included studies were provided in Table 1. Among the included trials, STAMPEDE used a multi-arm, multi-stage platform design to test efficacy of various combination treatments and shared one control arm. Five trials allowed maximal androgen blockade $(7,8,11,14,18,20)$. The median followup ranged from 14.4 to 138 months. All patients included were diagnosed as mHSPC, while men in LATITUDE trial were considered with high-risk disease which was defined as with at 
TABLE 1 | Characteristics of the included studies.

\begin{tabular}{|c|c|c|c|c|c|c|c|}
\hline Trial & $\begin{array}{l}\text { Study } \\
\text { design }\end{array}$ & Recruitment period & Population & $\begin{array}{l}\text { Median } \\
\text { follow-up } \\
\text { (month) }\end{array}$ & Treatment & $\begin{array}{l}\text { Sample } \\
\text { size }\end{array}$ & $\begin{array}{l}\text { Definition of } \\
\text { FFS }\end{array}$ \\
\hline & & & & & ADT & $724^{\#}$ & \\
\hline & & & & & ADT & $502^{\#}$ & \\
\hline GETUG-AFU15 & $\mathrm{RCT}$ & $\begin{array}{l}\text { October 2004-December } \\
2008\end{array}$ & $\mathrm{mHSPC}$ & 84 & $\begin{array}{l}\mathrm{ADT}^{*}+\text { Doc }(75 \mathrm{mg} / \mathrm{m} 2 \text { every } 3 \\
\text { weeks up to } 9 \text { cycles })+ \\
\text { corticosteroid premedication } \\
\text { before, on the day, and day after } \\
\text { infusion }\end{array}$ & 192 & $\begin{array}{l}\text { Time from randomization to } \\
\text { biochemical progression or } \\
\text { radiographic progression or death. }\end{array}$ \\
\hline & & & & & $\mathrm{ADT}^{*}$ & 393 & \\
\hline \multirow[t]{2}{*}{ LATITUDE } & $\mathrm{RCT}$ & $\begin{array}{l}\text { February 2013-December } \\
2014\end{array}$ & $\begin{array}{l}\text { High-risk } \\
\text { mHSPC }\end{array}$ & 30.4 & $\begin{array}{l}\mathrm{ADT}+\mathrm{Abi}(1,000 \mathrm{mg} \text { daily })+ \\
\text { prednisone ( } 5 \mathrm{mg} \text { daily })\end{array}$ & 597 & $\begin{array}{l}\text { Time from randomization to } \\
\text { radiographic progression or death } \\
\text { from any cause }\end{array}$ \\
\hline & & & & & ADT & 602 & \\
\hline ENZAMET & $\mathrm{RCT}$ & March 2014-2017 & $\mathrm{mHSPC}$ & 34 & ADT $^{\star}+$ Enza (160 mg daily) & 563 & $\begin{array}{l}\text { Time from randomization to } \\
\text { biochemical progression, clinical } \\
\text { progression, death from any cause, } \\
\text { or the last follow-up }\end{array}$ \\
\hline & & & & & $\mathrm{ADT}^{*}$ & 576 & \\
\hline \multirow[t]{2}{*}{ ZAPCA } & $\mathrm{RCT}$ & May 2008-December 2010 & mHSPC & 41.5 & $\begin{array}{l}\mathrm{ADT}^{*}+\text { Bis (ZA } 4 \mathrm{mg} \text { every } 4 \\
\text { weeks for up to } 2 \text { years) }\end{array}$ & 109 & $\begin{array}{l}\text { Time from randomization to } \\
\text { biochemical progression, clinical } \\
\text { progression, first SRE, death for any } \\
\text { reason }\end{array}$ \\
\hline & & & & & $\mathrm{ADT}^{*}$ & 110 & \\
\hline \multirow[t]{2}{*}{ STAMPEDE-Bis } & $\mathrm{RCT}$ & October 2005-March 2013 & mHSPC & 43 & $\begin{array}{l}\text { ADT + Bis (ZA } 4 \text { mg every } 3 \\
\text { weeks for } 6 \text { cycles, then every } 4 \\
\text { weeks until } 2 \text { years) }\end{array}$ & $366^{\#}$ & $\begin{array}{l}\text { Time from randomization to } \\
\text { biochemical failure, local/distant } \\
\text { metastases or death from } \mathrm{PCa}\end{array}$ \\
\hline & & & & & ADT & $724^{\#}$ & \\
\hline \multirow[t]{2}{*}{$\begin{array}{l}\text { STAMPEDE- } \\
\text { Doc+Bis }\end{array}$} & $\mathrm{RCT}$ & October 2005-March 2013 & $\mathrm{mHSPC}$ & 43 & $\begin{array}{l}\text { ADT + Bis (ZA } 4 \text { mg every } 3 \\
\text { weeks for } 6 \text { cycles, then every } 4 \\
\text { weeks until } 2 \text { years) + Doc ( } 75 \\
\mathrm{mg} / \mathrm{m}^{2} \text { every } 3 \text { weeks for } 6 \\
\text { cycles) + prednisolone ( } 10 \mathrm{mg} \\
\text { daily) }\end{array}$ & $365^{\#}$ & $\begin{array}{l}\text { Time from randomization to } \\
\text { biochemical failure, local/distant } \\
\text { metastases, or death from PCa }\end{array}$ \\
\hline & & & & & ADT & $724^{\#}$ & \\
\hline
\end{tabular}


TABLE 1 | Continued

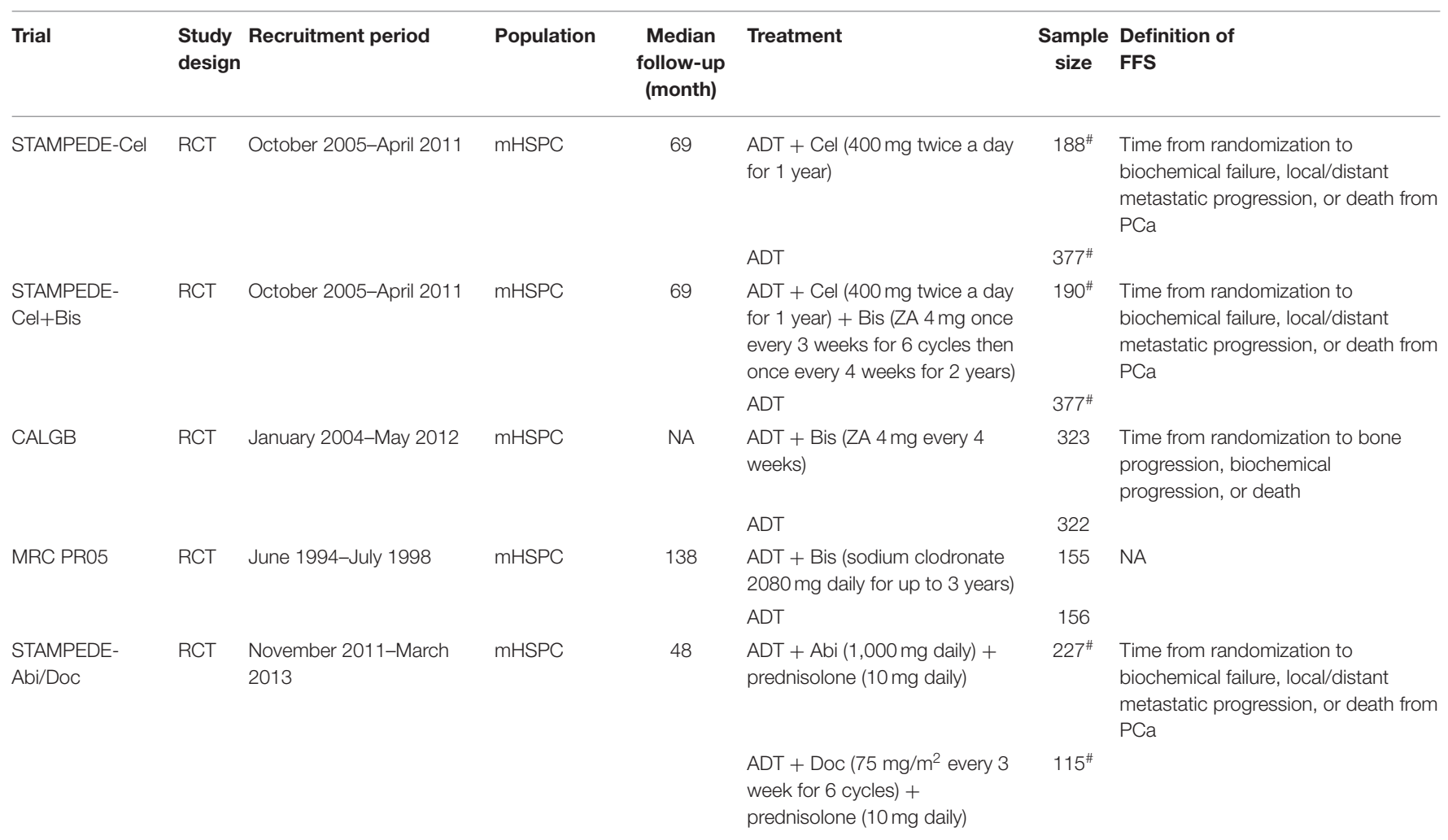

RCT, randomized controlled trial; $m$ HSPC, metastatic hormone-sensitive prostate cancer; Doc, docetaxel; Abi, abiraterone; Enza, enzalutamide; Apa, apalutamide; Bis, bisphosphonate; Cel, celecoxib; ZA, zoledronic acid; ADT, androgen-deprivation therapy; PCa, prostate cancer. NA, not applicable.

*Maximal androgen blockage allowed.

\#Data of metastatic group were used.

least two of the three following factors: Gleason score $\geq 8$, bone lesions $\geq 3$, and visceral metastasis (6). A majority of the patients had a favorable performance status and high Gleason score $(\geq 8)$. Detailed baseline characteristics of the included patients were summarized in Table 2. The assessment of the risk of bias for included studies was shown in Supplementary Figure 1.

\section{Direct Comparisons}

As shown in Figures 2A,B, ADT in addition to docetaxel $(\mathrm{HR}=0.74,95 \% \mathrm{CI}=0.61-0.90)$, abiraterone $(\mathrm{HR}=0.62$, $95 \% \mathrm{CI}=0.53-0.71)$, enzalutamide $(\mathrm{HR}=0.65,95 \% \mathrm{CI}=0.43-$ $0.98)$, apalutamide $(\mathrm{HR}=0.63,95 \% \mathrm{CI}=0.47-0.84)$, docetaxel + bisphosphonate $(\mathrm{HR}=0.79$, 95\% CI $=0.66-$ $0.95)$, bisphosphonate $(\mathrm{HR}=0.78,95 \% \mathrm{CI}=0.77-0.98)$, or bisphosphonate + celecoxib $(\mathrm{HR}=0.78,95 \% \mathrm{CI}=0.62-0.98)$ showed benefit in OS compared to ADT alone in patients with mHSPC. However, celecoxib plus ADT could not improve OS compared to $\mathrm{ADT}$ alone $(\mathrm{HR}=0.94,95 \% \mathrm{CI}=0.75-1.18)$. Moreover, abiraterone plus ADT showed no superiority than docetaxel plus ADT in men with $\mathrm{mHSPC}$ with a HR of 1.13 $(95 \% \mathrm{CI}=0.77-1.66)$.

In terms of FFS (Figures 2C,D), additional docetaxel $(\mathrm{HR}=0.62,95 \% \mathrm{CI}=0.56-0.69)$, abiraterone $(\mathrm{HR}=0.38$, $95 \% \mathrm{CI}=0.25-0.57)$ enzalutamide $(\mathrm{HR}=0.36$,
$95 \% \mathrm{CI}=0.30-0.44)$, apalutamide $(\mathrm{HR}=0.49,95 \% \mathrm{CI}=0.39$ $0.62)$, or docetaxel + bisphosphonate $(\mathrm{HR}=0.60,95 \% \mathrm{CI}=0.52$

0.69 ) showed strong benefits compared to ADT alone in men with $\mathrm{mHSPC}$. Besides, additional bisphosphonate $(\mathrm{HR}=0.87$, $95 \% \mathrm{CI}=0.79-0.96)$ or bisphosphonate + celecoxib $(\mathrm{HR}=0.77$, $95 \% \mathrm{CI}=0.63-0.94)$ showed moderate benefits compared to ADT alone. No statistically significant improvement in FFS was observed with additional celecoxib $(\mathrm{HR}=0.8695 \% \mathrm{CI}=0.79$ 1.04). Obvious net benefit was seen with abiraterone plus ADT $(\mathrm{HR}=0.56,95 \% \mathrm{CI}=0.42-0.75)$ compared to docetaxel plus ADT.

\section{Network Comparisons}

As shown in Figures 3, 4A, ADT in addition to docetaxel $(\mathrm{HR}=0.72,95 \% \mathrm{CrI}=0.60-0.86)$, abiraterone $(\mathrm{HR}=0.64$, $95 \% \mathrm{CrI}=0.53-0.80)$, enzalutamide $(\mathrm{HR}=0.64,95 \% \mathrm{CrI}=0.46-$ $0.88)$ or apalutamide $(\mathrm{HR}=0.63,95 \% \mathrm{CrI}=0.43-0.92)$ showed significant improvement in OS compared to ADT alone. While, according to the results of network meta-analysis, ADT with additional bisphosphonate $(\mathrm{HR}=0.86,95 \% \mathrm{CrI}=0.72-1.00)$, docetaxel + bisphosphonate $(\mathrm{HR}=0.79,95 \% \mathrm{CrI}=0.58-1.10)$, celecoxib $(\mathrm{HR}=0.94,95 \% \mathrm{CrI}=0.67-1.30)$, or bisphosphonate + celecoxib $(\mathrm{HR}=0.78,95 \% \mathrm{CrI}=0.56-1.10)$ showed no survival benefit compared to ADT alone. No one combination 


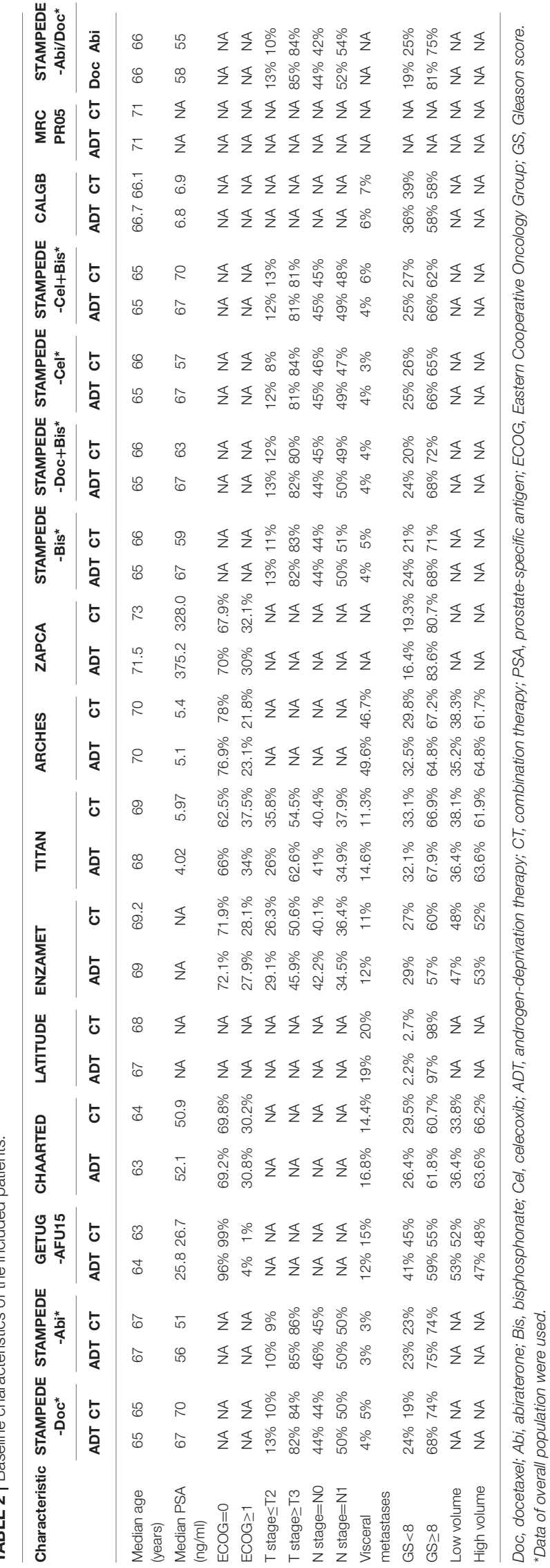

therapy showed significant superiority than another in terms of OS.

For FFS (Figures 3, 4B), obvious survival benefit was associated with $\mathrm{ADT}$ in addition to abiraterone $(\mathrm{HR}=0.37$, $95 \% \mathrm{CrI}=0.30-0.46)$, enzalutamide $(\mathrm{HR}=0.36,95 \% \mathrm{CrI}=0.28-$ $0.48)$, or apalutamide $(\mathrm{HR}=0.49,95 \% \mathrm{CrI}=0.35-0.70)$. Moderate benefit was observed with combination of ADT plus docetaxel or docetaxel + bisphosphonate $(\mathrm{HR}=0.60$, $95 \% \mathrm{CrI}=0.44-0.82)$. No significant benefit was seen with ADT in addition to bisphosphonate $(\mathrm{HR}=0.86,95 \% \mathrm{CrI}=0.71-1.00)$, celecoxib ( $\mathrm{HR}=0.86,95 \% \mathrm{CrI}=0.62-1.20)$ or bisphosphonate + celecoxib $(\mathrm{HR}=0.77,95 \% \mathrm{CrI}=0.55-1.10)$ compared to ADT alone. ADT with additional abiraterone or enzalutamide showed superiority over other combination therapies except for ADT plus apalutamide.

\section{Treatment Rankings}

As shown in Figure 4A, ADT plus abiraterone, enzalutamide or apalutamide seemed to be the three most effective treatments with a possibility of $21,32,37 \%$ ranking first, respectively, and a SUCRA of 80,79 , and $79 \%$, respectively, in terms of OS. As for FFS (Figure 4B), ADT plus abiraterone or enzalutamide seemed to be the most effective treatments with a probability of 42 and 54\% ranking first, respectively, and a SUCRA of 92 and $93 \%$, respectively.

\section{Subgroup Analysis for the Four Effective Therapies}

For patients with high-volume disease, abiraterone in addition with ADT seemed to be the most effective therapy with a probability of $46 \%$ ranking first and a SUCRA of $76 \%$ in terms of OS and 71 and $88 \%$, respectively, in terms of FFS (Figures 5A,B). For those with low-volume disease, enzalutamide plus ADT showed superiority to other therapies with a probability of $59 \%$ ranking first and a SUCRA of $82 \%$ in terms of OS (Figure 5C). While, in terms of FFS, additional abiraterone to ADT outperformed others with a probability of $47 \%$ ranking first and a SUCRA of 79\% (Figure 5D).

For men with visceral metastasis, abiraterone plus ADT seemed to be the best option with a probability of $46 \%$ ranking first and a SUCRA of $80 \%$ in terms of OS and $47 \%$ and $74 \%$, respectively, in terms of FFS (Figures 5E,F). For men without visceral metastasis, enzalutamide plus ADT was superior to other regimens with a probability of $29 \%$ ranking first and a SUCRA of $64 \%$ in terms of OS and $61 \%$ and $83 \%$, respectively, in terms of FFS (Figures 5G,H).

\section{Adverse Events}

Among the four effective treatments, docetaxel plus ADT and abiraterone plus ADT had the highest risk of overall AEs $\geq$ grade 3 with the same SUCRA of 79\%. Docetaxel in addition to ADT had the highest risk of febrile neutropenia and anemia with a SUCRA of 93 and 87\%, respectively. Abiraterone plus ADT had the highest risk of hypertension, cardiac disorder, and alanine transaminase (ALT) increase with a SUCRA of 90, 93, and $83 \%$, respectively. Apalutamide plus ADT was associated with the highest risk of fracture with a SUCRA of $65 \%$. ADT 


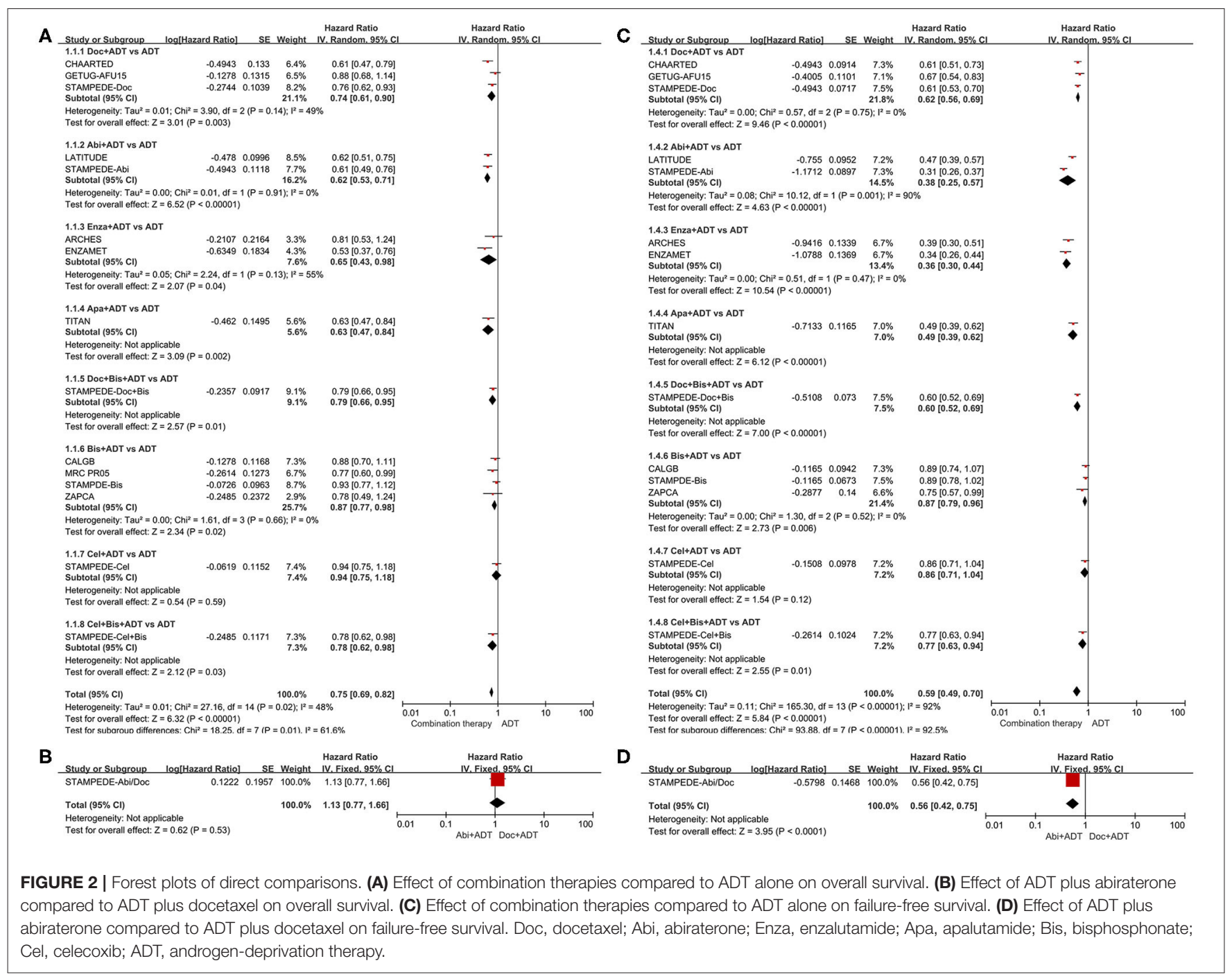

\begin{tabular}{|c|c|c|c|c|c|c|c|c|}
\hline Doc+ADT & $1.10(0.85-1.40)$ & $1.10(0.78-1.60)$ & $1.10(0.74-1.70)$ & $0.84(0.65-1.10)$ & $0.91(0.63-1.30)$ & $0.76(0.52-1.10)$ & $0.92(0.62-1.30)$ & $0.72(0.60-0.86)$ \\
\hline $0.59(0.46-0.74)$ & Abi+ADT & $1.00(0.70-1.50)$ & $1.00(0.67-1.60)$ & $0.75(0.58-1.00)$ & $0.82(0.57-1.20)$ & $0.69(0.47-1.00)$ & $0.83(0.57-1.20)$ & $0.64(0.53-0.80)$ \\
\hline $0.58(0.42-0.79)$ & $0.98(0.70-1.40)$ & Enza+ADT & $1.00(0.61-1.70)$ & $0.74(0.51-1.10)$ & $0.80(0.52-1.30)$ & $0.68(0.43-1.10)$ & $0.82(0.52-1.30)$ & $0.64(0.46-0.88)$ \\
\hline $0.78(0.52-1.2)$ & $1.30(0.88-2.00)$ & $1.30(0.86-2.10)$ & Apa+ADT & $0.74(0.49-1.10)$ & $0.80(0.49-1.30)$ & $0.67(0.41-1.10)$ & $0.81(0.49-1.40)$ & $0.63(0.43-0.92)$ \\
\hline $1.40(1.00-1.70)$ & $2.30(1.70-3.00)$ & $2.40(1.70-3.20)$ & $1.80(1.20-2.60)$ & Bis+ADT & $1.10(0.76-1.50)$ & $0.91(0.62-1.30)$ & $1.10(0.75-1.60)$ & $0.86(0.72-1.00)$ \\
\hline $0.95(0.66-1.30)$ & $1.60(1.10-2.30)$ & $1.60(1.10-2.50)$ & $1.20(0.76-1.90)$ & $0.70(0.49-1.00)$ & Bis+Doc+ADT & $0.84(0.53-1.30)$ & $1.00(0.64-1.60)$ & $0.79(0.58-1.10)$ \\
\hline $1.40(0.93-2.00)$ & $2.30(1.60-3.40)$ & $2.40(1.60-3.60)$ & $1.80(1.10-2.80)$ & $1.00(0.69-1.50)$ & $1.40(0.91-2.30)$ & Cel+ADT & $1.20(0.76-1.90)$ & $0.94(0.67-1.30)$ \\
\hline $1.20(0.83-1.80)$ & $2.10(1.40-3.00)$ & $2.10(1.40-3.20)$ & $1.60(0.96-2.50)$ & $0.90(0.61-1.30)$ & $1.30(0.81-2.00)$ & $0.89(0.55-1.40)$ & Bis+Cel+ADT & $0.78(0.56-1.10)$ \\
\hline $1.60(1.30-1.90)$ & $2.70(2.20-3.30)$ & $2.70(2.10-3.60)$ & $2.00(1.40-2.90)$ & $1.20(0.97-1.40)$ & $1.70(1.20-2.30)$ & $1.20(0.83-1.60)$ & $1.30(0.93-1.80)$ & ADT \\
\hline
\end{tabular}

FIGURE 3 | Network comparisons of effect of systemic therapies on overall survival (yellow) and failure-free survival (blue). Results are the HRs with 95\% Crls from the network meta-analysis between the row intervention and column intervention. Doc, docetaxel; Abi, abiraterone; Enza, enzalutamide; Apa, apalutamide; Bis, bisphosphonate; Cel, celecoxib; ADT, androgen-deprivation therapy.

in addition with docetaxel, abiraterone or enzalutamide had a similar risk of nervous system disorder (SUCRA: 65, 62, and 65\%, respectively). On the contrary, apalutamide plus ADT had the lowest risk of overall AEs, anemia and hypertension (SUCRA:
28, 25, and 24\%, respectively). Abiraterone plus ADT had the lowest risk of fracture with a SUCRA of $42 \%$. Enzalutamide plus ADT had the lowest risk of febrile neutropenia and ALT increase (SUCRA: 33 and 29\%, respectively). Docetaxel plus 
A

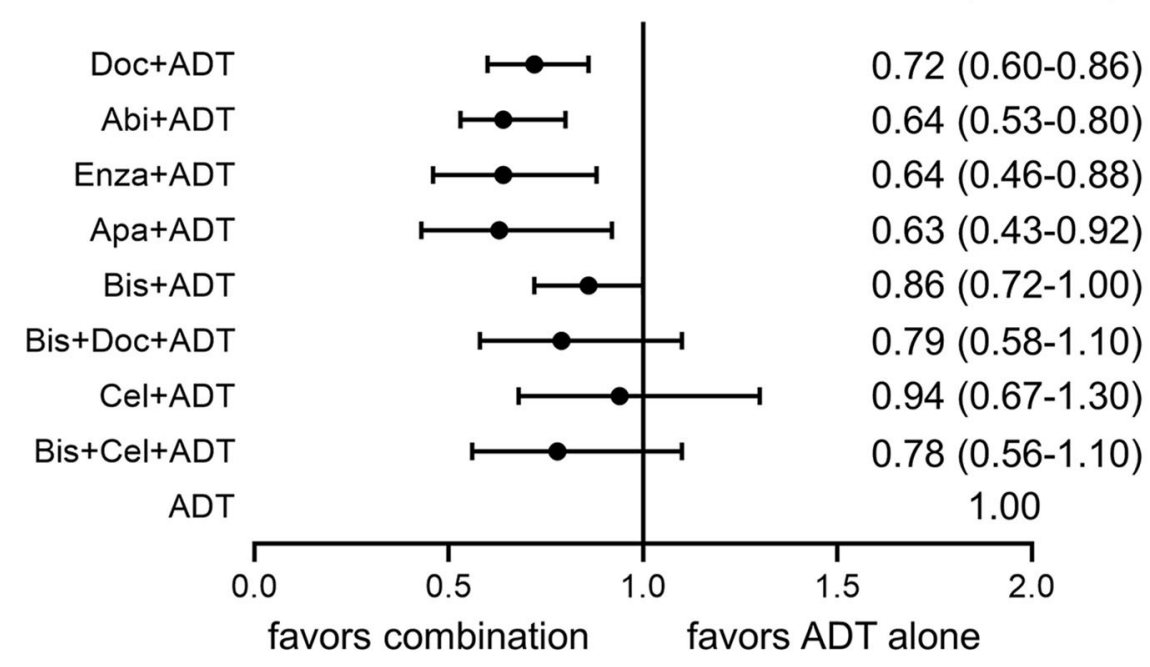

B

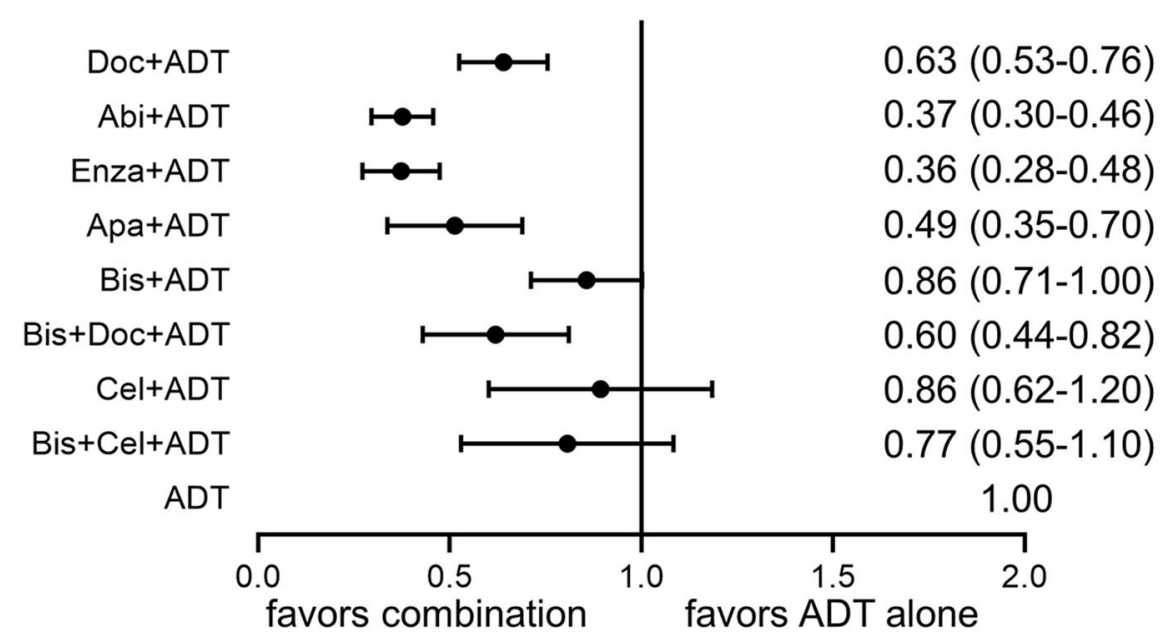

\section{favors combination favors ADT alone}

Probability of ranking first

$3 \%$

$21 \%$

$32 \%$

$37 \%$

$0 \%$

$3 \%$

$1 \%$

$4 \%$

$0 \%$
SUCRA

$62 \%$

$80 \%$

$79 \%$

$79 \%$

$31 \%$

$45 \%$

$19 \%$

$48 \%$

$6 \%$

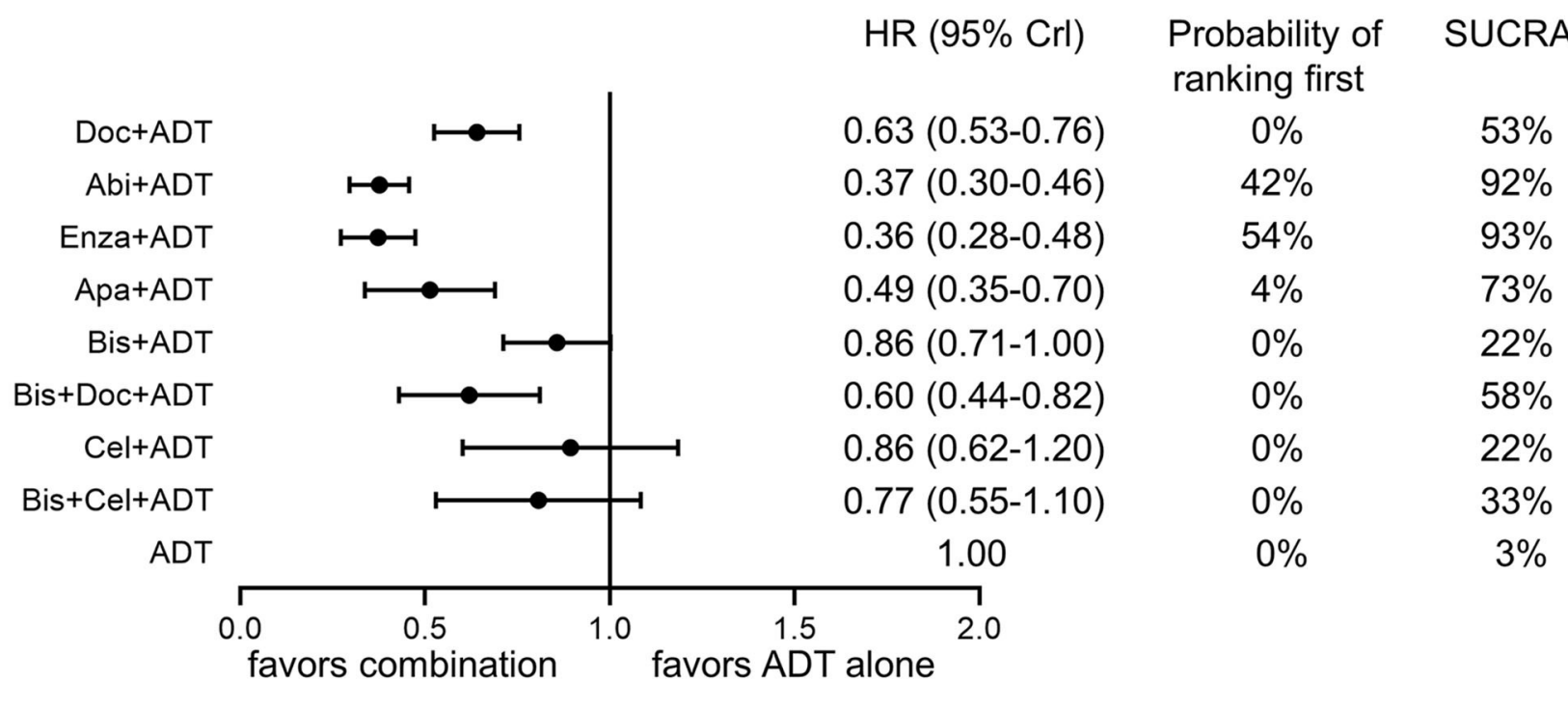

FIGURE 4 | Forest plots of network comparisons of effect of combination therapies compared to ADT alone on overall survival (A) and failure-free survival (B). Rankings are presented as probabilities of ranking first and SUCRA. Doc, docetaxel; Abi, abiraterone; Enza, enzalutamide; Apa, apalutamide; ADT, androgen-deprivation therapy.

ADT had the lowest risk of cardiac disorder with a SUCRA of $26 \%$.

\section{DISCUSSION}

Several previous meta-analyses have indirectly compared various systemic therapies in terms of survival benefit in mHSPC patients $(16,17,24)$. Recently, ADT in combination with novel ARtargeted therapies including enzalutamide and apalutamide has been reported to improve survival in patients with $\mathrm{mHSPC}$, which provided extra effective systemic treatment choices for this population. The present network meta-analysis comprehensively evaluated the efficacy of current systemic therapies for men with mHSPC based on the most up-to-date results from RCTs.
We found that ADT in addition to docetaxel, abiraterone, enzalutamide or apalutamide could significantly prolong both FFS and OS compared to ADT alone in men with mHSPC and abiraterone and enzalutamide appeared to be the most effective treatments for this setting.

Our results were in consistence with previous studies which reported the superiority of docetaxel and abiraterone to standard ADT in patients with $\operatorname{mHSPC}(16,17,24,25)$. However, whether abiraterone is better than docetaxel in this population remains a debate. Recently, the STAMPEDE trial directly compared ADT plus docetaxel and ADT plus abiraterone in men with $\mathrm{mHSPC}$ based on its multi-arm design and showed an advantage of ADT plus abiraterone on FFS but not OS compared to ADT plus docetaxel (15). This result should be interpreted cautiously, 


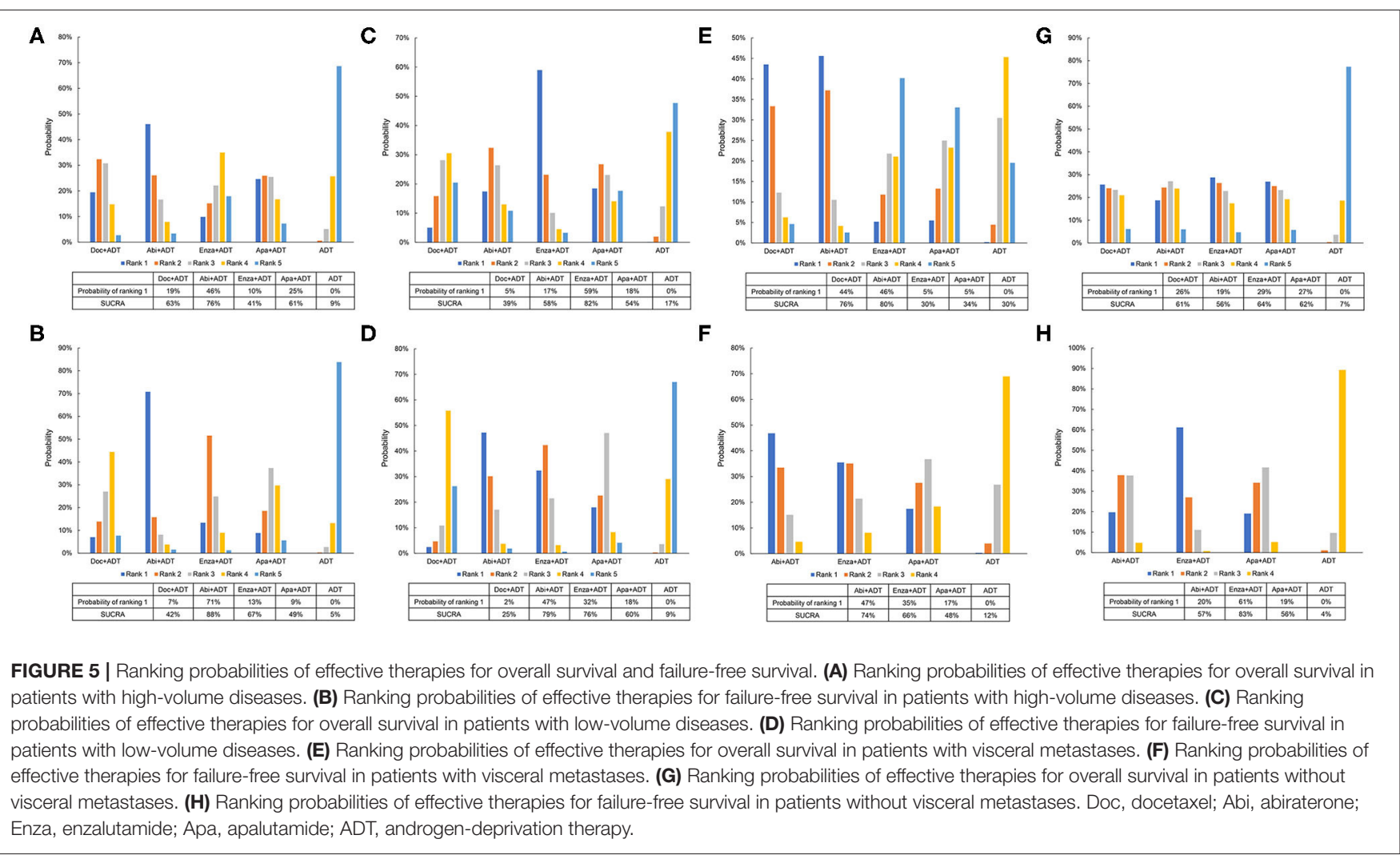

as the trial was not designed to directly compare these two therapies and might be underpowered to detect the differences. Our findings also indicated that patients with mHSPC could benefit more from ADT in combination with abiraterone with respect to FFS, but had similar OS with those received ADT plus docetaxel. Network meta-analysis is considered to be more powerful in detecting differences between treatments because of more patients included and the combination of direct and indirect comparisons. However, it should be noted that the differences in patient baseline characteristics might have an impact on the results of comparisons. The sample size of the GETUG-AFU15 trial was relatively small and approximately half of the patients had low-risk diseases according to Glass risk stratification or low-volume diseases based on CHAARTED criteria, which might attenuate the effects of docetaxel (8). While, as mentioned previously, patients in the LATITUDE trial all had high-risk diseases, which might inversely overestimate the benefit of abiraterone (6). Therefore, SUCRA analysis demonstrated that abiraterone had a higher probability to be the preferred treatment than docetaxel despite the lack of statistical significance in network comparison. Post-hoc analyses of the current RCTs based on consistent stratification criteria and results of direct comparisons from clinical trials are needed to provide more reliable evidence.

Enzalutamide and apalutamide are novel potent AR-targeted agents and have been proved their efficacy in mHSPC setting by recent RCTs (18-20). To the best of our knowledge, this is the first network meta-analysis comparing enzalutamide and apalutamide with other systemic combination regimens in men harboring $\mathrm{mHSPC}$. The results suggested that both enzalutamide and apalutamide could significantly improve FFS as well as OS compared to ADT alone. Moreover, enzalutamide and apalutamide had similar efficacy as abiraterone over other regimens based on SUCRA analysis. The ENZAMET trial allowed early use of docetaxel and showed significant improvement in survival in overall population and patients without early docetaxel but limited effects on survival in patients with early docetaxel (20). In our network meta-analysis, given the established role of docetaxel in the management of mHSPC, we extracted data from subgroup without early docetaxel to preclude the influence of docetaxel and better compare the effects of enzalutamide with other therapies. The benefit of ADT in addition with enzalutamide was also confirmed in the ARCHES trial (18). However, due to relatively short follow-up time, no statistically significant improvement in OS was observed in the ARCHES trial.

The extent of disease has a strong association with the prognosis of patients and could result in different responses to therapies (14). Therefore, selection of candidates based on disease extent to receive proper treatment is essential to reach maximal benefits. Despite similar efficacy of abiraterone and enzalutamide for overall mHSPC setting, the subgroup analyses in the present study suggested the superiority of abiraterone in addition with ADT in patients with high-volume disease or visceral metastasis and enzalutamide plus ADT in patients with low-volume disease or without visceral metastasis. 
Compared to other trials, the two trials (ENZAMET and ARCHES) on enzalutamide seemed to be complicated with more confounding factors such as prior and concomitant use of docetaxel, various standard nonsteroidal antiandrogen drugs and a high proportion of metachronous metastatic PCa (18, 20). Previous studies have demonstrated that different standard nonsteroidal antiandrogens had varied impacts on prognosis and metachronous metastatic PCa had poorer response to chemohormonal therapy and shorter survival than de novo metastatic PCa, which might partially explain the differences in survival benefit of enzalutamide on patients with different extensive diseases (26-28).

Making the optimal treatment decision is not only about pursuing maximal survival benefit but also decreasing AEs. According to our analyses, additional docetaxel and abiraterone were associated with a higher risk of overall AEs. Additional docetaxel was associated with a higher risk of myelosuppression, abiraterone had a higher risk of hypertension, cardiac disorder and ALT increase, and apalutamide had a higher risk of fracture. Patients in the LATITUDE trial had a higher incidence of AEs than those in other trials, which might result from poorer baseline characteristics (6). These results could help clinicians select therapies for patients under specific physical conditions to reduce and even avoid some treatment-induced AEs. Moreover, enzalutamide and apalutamide require no glucocorticoid which might be safer in patients with poorly controlled diabetes or heart failure.

This network meta-analysis is not without limitations. First, heterogeneities existed in the baseline characteristics of the include trials and patients. Maximal androgen blockage was allowed in five trials and prior or concomitant use of docetaxel in three trials. Besides, as shown in Table 1, several trials have different definitions of FFS with others and reported biochemical and radiographic progression separately. Also, the definition of high-volume disease in the TITAN trial was a little bit different from CHAARTED criteria. Third, fewer studies and smaller sample sizes in subgroup analysis could attenuate the power to detect differences in survival and increase bias. Fourth, the STAMPEDE trial used a multi-arm, multi-stage platform design, in which combination therapy groups were compared with the same control group. Moreover, due to limited comparisons and data on AEs, some of the results might differ from the real world. It is worth noting that the cardiotoxicity of docetaxel is not rare in spite of the lowest risk according to our results (29). Large prospective trials are needed to provide more potent evidence.

\section{REFERENCES}

1. Siegel RL, Miller KD, Jemal A. Cancer statistics, 2019. CA Cancer J Clin. (2019) 69:7-34. doi: 10.3322/caac.21551

2. Eisenberger MA, Blumenstein BA, Crawford ED, Miller G, McLeod DG, Loehrer PJ, et al. Bilateral orchiectomy with or without flutamide for metastatic prostate cancer. $N$ Engl J Med. (1998) 339:1036-42. doi: 10.1056/NEJM199810083391504

3. Wu JN, Fish KM, Evans CP, Devere White RW, Dall'Era MA. No improvement noted in overall or cause-specific survival for men presenting

\section{CONCLUSION}

ADT with additional docetaxel, abiraterone, enzalutamide, or apalutamide could significantly improve survival compared to ADT alone in patients with mHSPC. ADT plus abiraterone or enzalutamide appeared to be the most effective treatments for overall population. Abiraterone plus ADT showed superiority in patients with high-volume disease or visceral metastasis and enzalutamide plus ADT outperformed other treatments in patients with low-volume disease or without visceral metastasis. Additional docetaxel and abiraterone were associated with a higher risk of AEs. Clinicians should balance the efficacy, safety, and even the extent of disease to select the optimal treatment.

\section{DATA AVAILABILITY STATEMENT}

All datasets analyzed for this study are included in the article and the supplementary files.

\section{AUTHOR CONTRIBUTIONS}

JC and YN designed the study, analyzed the data, and wrote the manuscript. BL and GS helped literature searching and data analysis. SZ, XZ, and ZW helped prepare materials and revise the manuscript. PS and HZ supervised the project and revised the manuscript. All authors read and approved the final manuscript. All authors contributed to the article and approved the submitted version.

\section{FUNDING}

This study was supported by grants from National Natural Science Foundation of China (NSFC 81402110, 81672547, and 81272820 ) and 1.3.5 project for disciplines of excellence, West China Hospital, Sichuan University.

\section{SUPPLEMENTARY MATERIAL}

The Supplementary Material for this article can be found online at: https://www.frontiersin.org/articles/10.3389/fonc. 2020.519388/full\#supplementary-material

Supplementary Figure 1 | Assessment of risk of bias of the included studies. with metastatic prostate cancer over a 20-year period. Cancer. (2014) 120:81823. doi: $10.1002 /$ cncr.28485

4. Dearnaley DP, Sydes MR, Mason MD, Stott M, Powell CS, Robinson ACR, et al. A double-blind, placebo-controlled, randomized trial of oral sodium clodronate for metastatic prostate cancer (MRC PR05 Trial). J Natl Cancer Inst. (2003) 95:1300-11. doi: 10.1093/jnci/dj g038

5. Dearnaley DP, Mason MD, Parmar MKB, Sanders K, Sydes MR. Adjuvant therapy with oral sodium clodronate in locally advanced and metastatic prostate cancer: long-term overall survival results from the MRC PR04 
and PR05 randomised controlled trials. Lancet Oncol. (2009) 10:8726. doi: 10.1016/S1470-2045(09)70201-3

6. Fizazi K, Tran N, Fein L, Matsubara N, Rodriguez-Antolin A, Alekseev BY, et al. Abiraterone plus prednisone in metastatic, castration-sensitive prostate cancer. N Engl J Med. (2017) 377:352-60. doi: 10.1056/NEJMoa1704174

7. Gravis G, Boher JM, Joly F, Soulie M, Albiges L, Priou F, et al. Androgen deprivation therapy (ADT) plus docetaxel versus $\mathrm{ADT}$ alone in metastatic non castrate prostate cancer: impact of metastatic burden and long-term survival analysis of the randomized phase 3 GETUG-AFU15 trial. Eur Urol. (2016) 70:256-62. doi: 10.1016/j.eururo.2015.11.005

8. Gravis G, Fizazi K, Joly F, Oudard S, Priou F, Esterni B, et al. Androgendeprivation therapy alone or with docetaxel in non-castrate metastatic prostate cancer (GETUG-AFU 15): a randomised, open-label, phase 3 trial. Lancet Oncol. (2013) 14:149-58. doi: 10.1016/S1470-2045(12)70560-0

9. James ND, de Bono JS, Spears MR, Clarke NW, Mason MD, Dearnaley DP, et al. Abiraterone for prostate cancer not previously treated with hormone therapy. N Engl J Med. (2017) 377:338-51. doi: 10.1056/NEJMoa1702900

10. James ND, Sydes MR, Clarke NW, Mason MD, Dearnaley DP, Spears MR, et al. Addition of docetaxel, zoledronic acid, or both to first-line long-term hormone therapy in prostate cancer (STAMPEDE): survival results from an adaptive, multiarm, multistage, platform randomised controlled trial. Lancet. (2016) 387:1163-77. doi: 10.1016/S0140-6736(15)01037-5

11. Kamba T, Kamoto T, Maruo S, Kikuchi T, Shimizu Y, Namiki S, et al. A phase III multicenter, randomized, controlled study of combined androgen blockade with versus without zoledronic acid in prostate cancer patients with metastatic bone disease: results of the ZAPCA trial. Int J Clin Oncol. (2017) 22:166-73. doi: 10.1007/s10147-016-1037-2

12. Mason MD, Clarke NW, James ND, Dearnaley DP, Spears MR, Ritchie AWS, et al. Adding celecoxib with or without zoledronic acid for hormonenaïve prostate cancer: long-term survival results from an adaptive, multiarm, multistage, platform, randomized controlled trial. J Clin Oncol. (2017) 35:1530-41. doi: 10.1200/JCO.2016.69.0677

13. Smith MR, Halabi S, Ryan CJ, Hussain A, Vogelzang N, Stadler W, et al. Randomized controlled trial of early zoledronic acid in men with castrationsensitive prostate cancer and bone metastases: results of CALGB 90202 (alliance). J Clin Oncol. (2014) 32:1143-50. doi: 10.1200/JCO.2013.51.6500

14. Sweeney CJ, Chen YH, Carducci M, Liu G, Jarrard DF, Eisenberger M, et al. chemohormonal therapy in metastatic hormone-sensitive prostate cancer. $N$ Engl J Med. (2015) 373:737-46. doi: 10.1056/NEJMoa1503747

15. Sydes MR, Spears MR, Mason MD, Clarke NW, Dearnaley DP, de Bono JS, et al. Adding abiraterone or docetaxel to long-term hormone therapy for prostate cancer: directly randomised data from the STAMPEDE multi-arm, multi-stage platform protocol. Ann Oncol. (2018) 29:1235-48.

16. Tan PS, Aguiar P Jr, Haaland B, Lopes G. Addition of abiraterone, docetaxel, bisphosphonate, celecoxib or combinations to androgen-deprivation therapy (ADT) for metastatic hormone-sensitive prostate cancer (mHSPC): a network meta-analysis. Prostate Cancer Prostatic Dis. (2018) 21:51623. doi: 10.1038/s41391-018-0055-8

17. Vale CL, Fisher DJ, White IR, Carpenter JR, Burdett S, Clarke NW, et al. What is the optimal systemic treatment of men with metastatic, hormone-naive prostate cancer? A STOPCAP systematic review and network meta-analysis. Ann Oncol. (2018) 29:1249-57. doi: 10.1093/annonc/mdy071

18. Armstrong AJ, Szmulewitz RZ, Petrylak DP, Holzbeierlein J, Villers A, Azad A, et al. ARCHES: a randomized, phase iii study of androgen deprivation therapy with enzalutamide or placebo in men with metastatic hormone-sensitive prostate cancer. J Clin Oncol. (2019) 37:2974-86. doi: 10.1200/JCO.19.0 0799
19. Chi KN, Agarwal N, Bjartell A, Chung BH, Pereira de Santana Gomes AJ, Given R, et al. Apalutamide for metastatic, castration-sensitive prostate cancer. N Engl J Med. (2019) 381:13-24. doi: 10.1056/NEJMoa1903307

20. Davis ID, Martin AJ, Stockler MR, Begbie S, Chi KN, Chowdhury S, et al. Enzalutamide with standard first-line therapy in metastatic prostate cancer. N Engl J Med. (2019) 381:121-31. doi: 10.1056/NEJMoa1903835

21. Stewart LA, Clarke M, Rovers M, Riley RD, Simmonds M, Stewart G, et al. Preferred reporting items for systematic review and meta-analyses of individual participant data: the PRISMA-IPD statement. JAMA. (2015) 313:1657-65. doi: 10.1001/jama.2015.3656

22. Salanti G, Ades AE, Ioannidis JPA. Graphical methods and numerical summaries for presenting results from multiple-treatment meta-analysis: an overview and tutorial. J Clin Epidemiol. (2011) 64:163-71. doi: 10.1016/j.jclinepi.2010.03.016

23. Hoyle AP, Ali A, James ND, Cook A, Parker CC, de Bono JS, et al. Abiraterone in "high-" and "low-risk" metastatic hormone-sensitive prostate cancer. Eur Urol. (2019) 76:719-28. doi: 10.1016/j.eururo.2019.08.006

24. Sun G, Zhang X, Chen J, Liao B, Liu Z, Zhao J, et al. What kind of patients with castration-naive prostate cancer can benefit from upfront docetaxel and abiraterone: a systematic review and a network meta-analysis. Urol Oncol. (2018) 36:505-17. doi: 10.1016/j.urolonc.2018.09.005

25. Vale CL, Burdett S, Rydzewska LHM, Albiges L, Clarke NW, Fisher D, et al. Addition of docetaxel or bisphosphonates to standard of care in men with localised or metastatic, hormone-sensitive prostate cancer: a systematic review and meta-analyses of aggregate data. Lancet Oncol. (2016) 17:24356. doi: 10.1016/S1470-2045(15)00489-1

26. Kyriakopoulos CE, Chen YH, Carducci MA, Liu G, Jarrard DF, Hahn NM, et al. Chemohormonal therapy in metastatic hormonesensitive prostate cancer: long-term survival analysis of the randomized phase III E3805 CHAARTED trial. J Clin Oncol. (2018) 36:1080-7. doi: 10.1200/JCO.2017.75.3657

27. Schellhammer PF, Sharifi R, Block NL, Soloway MS, Venner PM, Patterson AL, et al. Clinical benefits of bicalutamide compared with flutamide in combined androgen blockade for patients with advanced prostatic carcinoma: final report of a double-blind, randomized, multicenter trial. Casodex Combination Study Group. Urology. (1997) 50:330-6. doi: 10.1016/S0090-4295(97)00279-3

28. Yokomizo Y, Kawahara T, Miyoshi Y, Otani M, Yamanaka S, Teranishi J, et al. Efficacy of immediate switching from bicalutamide to flutamide as second-line combined androgen blockade. Biomed Res Int. (2016) 2016:4083183. doi: $10.1155 / 2016 / 4083183$

29. Petrylak DP, Tangen CM, Hussain MH, Lara PN Jr, Jones JA, Taplin ME, et al. Docetaxel and estramustine compared with mitoxantrone and prednisone for advanced refractory prostate cancer. N Engl J Med. (2004) 351:151320. doi: 10.1056/NEJMoa041318

Conflict of Interest: The authors declare that the research was conducted in the absence of any commercial or financial relationships that could be construed as a potential conflict of interest.

Copyright $\odot 2020$ Chen, Ni, Sun, Liao, Zhang, Zhao, Zhu, Wang, Shen and Zeng. This is an open-access article distributed under the terms of the Creative Commons Attribution License (CC BY). The use, distribution or reproduction in other forums is permitted, provided the original author(s) and the copyright owner(s) are credited and that the original publication in this journal is cited, in accordance with accepted academic practice. No use, distribution or reproduction is permitted which does not comply with these terms. 